\title{
Cosmological neutrino entanglement and quantum pressure
}

\author{
D. Pfenniger and V. Muccione
}

Geneva Observatory, University of Geneva, 1290 Sauverny, Switzerland

Received 8 November 2005 / Accepted 3 June 2006

\begin{abstract}
Context. The widespread view that cosmological neutrinos, even if massive, are well described since the decoupling redshift $z \approx 10^{10}$ down to the present epoch by an almost perfectly collisionless fluid of classical point particles is re-examined.

Aims. In view of the likely sub-eV rest mass of neutrinos, the main effects due to their fermionic nature are studied.

Methods. By numerical means we calculate the accurate entropy, fugacity and pressure of cosmological neutrinos in the Universe expansion. By solving the Schrödinger equation we derive how and how fast semi-degenerate identical free fermions become entangled.

Results. We find that for sub-eV neutrinos the exchange degeneracy has significantly increased during the relativistic to nonrelativistic transition epoch at $z \approx 10^{4}-10^{5}$. At all times neutrinos become entangled in less than $10^{-6} \mathrm{~s}$, much faster than any plausible decoherence time. The total pressure is increased by quantum effect from 5\% at high redshifts to $68 \%$ at low redshifts with respect to a collisionless classical fluid.

Conclusions. The quantum overpressure has no dynamical consequences in the homogeneous regime at high redshifts, but must be significant for neutrino clustering during the non-linear structure formation epoch at low redshifts.
\end{abstract}

Key words. cosmology: theory - neutrions - cosmology: large-scale structure of Universe - cosmology: dark matter elementary particles

\section{Introduction}

We re-examine the common view in astronomy that cosmological neutrinos, if massive, are well described since their decoupling redshift $z \approx 10^{10}$ down to the present epoch by a collisionless fluid of classical particles (Primack \& Gross 2000). Neglected up to now is one of the most fundamental and well established principle of modern physics, the fermion/boson symmetrisation rules from which Pauli's exclusion principle follows (Pauli 1925; Dirac 1926; Fermi 1926).

When we asked cosmologists how massive neutrinos behave today despite their degeneracy ${ }^{1}$, they would argue that they behave as a collisionless fluid of classical particles. In cosmological $N$-body simulations (e.g., Klypin et al. 1993) the only difference between neutrinos and cold dark matter particles is the respective initial velocity dispersion.

When we asked theoretical and experimental physicists familiar with particle physics, quantum and statistical mechanics, they would find obvious that degeneracy pressure between neutrinos must be taken into account despite their negligible weak interaction cross section, because degeneracy pressure results from Pauli's principle and depends only on the exchange symmetry of identical particles. In the limit of negligible interaction in the particle Hamiltonian, the fermionic or bosonic behaviour remains, therefore the particle statistics is an aspect unrelated to the strength of the particle-particle interaction.

In summary, in the cosmologist view neutrinos are well described with a distribution of collisionless classical particles following the collisionless Boltzmann equation, while in the physicist view neutrinos are quantum particles, i.e., non-local

\footnotetext{
1 In the context of cosmological neutrinos, the word "degeneracy" is used with different meanings. Here it concerns the particle exchange degeneracy, not the mass or the chemical potential degeneracy.
}

entities, and when they are indistinguishable they behave collectively according to the well established principles of quantum statistics. In cosmological simulations neutrinos would be better represented by a collisional gas with the Fermi-Dirac equation of state.

This distinction has no consequence during the relativistic homogeneous regime because the initial Fermi-Dirac statistics is preserved by the Universe expansion (Weinberg 1972). But this is no longer true during the non-linear structure formation phase, where the different matter components induce inhomogeneous perturbations driving neutrinos out of thermal equilibrium. Then the degeneracy pressure is different from the straight kinetic pressure of collisionless particles, therefore the fate of massive neutrinos in galaxy clusters or galaxies differs if neutrinos behave as classical or quantum particles.

In the recent years this problem has taken importance for the description of the non-linear phase of structure formation, because the likely mass of neutrinos (Tegmark et al. 2005) determined in different experiments appear to sum up to at least the visible baryon mass fraction. If neutrinos behave collectively as a classical collisionless fluid, then their interaction with the other matter components is limited to the weak and gravitational interactions, while if neutrinos behave as a coherent Fermi gas, then they can develop shocks, increase entropy, and lead to nonlinear evolution as hard to predict as the baryon one during the structure formation phase.

The paradox raised by the cosmological neutrinos goes in fact at the heart of still debated fundamental open problems in quantum mechanics, such as the meaning of measurements and wave-function reductions, the destruction or built-up of coherence between particles, etc. It concerns also the problem of irreversibility in quantum systems, which, as in classical systems, are strictly time-reversible in theory, but dissipative in practice. 


\section{The classical description}

It has been usual in the past decades to assume neutrinos as either massless relativistic particles, or as a dark matter candidate able to account for a substantial part of the dark matter, which implies a neutrino rest mass well above $10 \mathrm{eV}$. The classical reasoning (e.g., Tremaine \& Gunn 1979; Peebles 1993) goes as follow. Neutrinos are generated above redshift $z \approx 10^{10}$ by elementary particle creation and destruction processes. The important point is that at these densities $\left(\sim 10^{31} \mathrm{~cm}^{-3}\right)$ and temperature $\left(\sim 10^{10} \mathrm{~K}\right)$ the electro-weak force cross-sections are large enough to make the reaction time-scales much shorter than the Universe expansion time-scale (see Weinberg 1972; Peebles 1993; Padmanabhan 2002). This ensures thermal equilibrium and well determined initial thermodynamical conditions. At redshift below about $z \approx 10^{10}$ the creation of electron-positron pairs, which releases the last neutrinos and anti-neutrinos, drops because the thermal energy of particles falls below the $511 \mathrm{keV}$ electron-positron rest mass energy. The neutrino mean free-path for weak interaction diverges and neutrinos are since then considered as propagating freely across the Universe, only subject to gravity perturbations.

The neutrino weak interaction cross section is exceedingly small (Peacock 1999):

$\sigma \sim 4 \times 10^{-64} T^{2} \mathrm{~cm}^{2}$.

Therefore the widespread view among cosmologists is to consider neutrinos as collisionless classical particles, i.e., well localised mass concentrations moving according to Newton's laws. Consequently, neutrinos are supposed to obey accurately the collisionless Boltzmann equation. The neutrinos follow an incompressible flow in phase space, even during the possibly complex non-linear structure formation phase. This leads to the well known phase space density constraint (Tremaine \& Gunn 1979). In this classical description violent relaxation processes dilute phase space irregularities into a coarse grained distribution, and neutrinos can not increase their degeneracy.

In the conventional description neutrinos are estimated insensitive to quantum effects. Peebles (1993, p. 445) argues that their de Broglie wavelength

$\lambda_{\mathrm{dB}}=\frac{h}{m_{\nu} v}$,

is a small fraction of $\mathrm{cm}$. The wavelength derived by Peebles, $0.001 \mathrm{~cm}$, is obtained supposing a velocity of $v=300 \mathrm{~km} \mathrm{~s}^{-1}$ and a rather large neutrino mass of $m_{v}=123 \mathrm{eV}$. Peebles' concludes: "classical physics gives an excellent approximation to the motion of neutrinos, and Liouville's theorem assures us the classical orbits will not violate the exclusion principle, because the occupation number [...] is conserved". The picture is thus that neutrinos are point masses regularly distributed in phase space, keeping approximately their relative phase space distances sufficiently large to allow neglecting Pauli's exclusion principle. A uniformly expanding flow of particles in space is a uniformly shearing flow in phase space. Nearby particles in phase space do see fluctuating neighbour distances. Therefore if any effect depends on phase space relative distances, phase space density perturbations can propagate.

Peebles invokes Pauli's exclusion principle and the de Broglie wavelength as criteria for neglecting or not quantum effects. Below we will see that relativistic and non-relativistic neutrinos are in fact rather degenerate from the beginning, so Pauli's principle matters.

\section{The quantum description}

\subsection{State of cosmological neutrinos}

Let us check Peebles' assumption with recent estimates of the neutrino properties (Dolgov 2002), keeping in mind that the classical world is only an approximation of the quantum world, and that the important point is not the ratio of scales (such as the de Broglie wavelength vs. the size of cosmic structures) but the ratio of the phase space occupation density to Planck's constant $h$ cubed.

In order to not complicate the discussion, we ignore here the effect of neutrino oscillations for which the proper collective treatment has been only recently worked out (Strack \& Burrows 2005). It suffices here to point out that instead of discussing the neutrino flavours (the electron, muon and tau neutrinos which are superpositions of mass states), we need to consider the pure mass states $\left(m_{1}, m_{2}\right.$, and $\left.m_{3}\right)$. The oscillations introduce coupling terms between the different mass states (Strack \& Burrows 2005), and therefore increase the coupling between neutrinos species.

Today's number density of electron neutrinos and antineutrinos issued from the Big Bang (Dolgov 2002; Peebles 1993, p. 163) of a single family is $3 / 11$ the number of cosmic background photons:

$n_{v, 0}=\frac{3}{11} n_{\gamma} \approx 112 \mathrm{~cm}^{-3}$.

Therefore the mean inter-particle distance $n_{v, 0}^{-1 / 3} \approx 0.2 \mathrm{~cm}$ is comparable to their de Broglie wavelength

$\lambda_{\mathrm{dB}} \approx 0.4 \mathrm{~cm}\left(\frac{v}{1000 \mathrm{~km} \mathrm{~s}^{-1}}\right)^{-1}\left(\frac{m_{v}}{0.1 \mathrm{eV}}\right)^{-1}$.

So with sub-eV rest mass neutrinos appear rather degenerate. Crucial is obviously the value of the assumed velocity. The chosen $v$ is reasonable for neutrinos bound inside galaxy clusters, but it is not obvious whether neutrinos could ever be trapped inside clusters. So let us consider the least favourable hypothesis that identical neutrinos are still homogeneously distributed, with a density of 112 or $56 \mathrm{~cm}^{-3}$ depending on the still unknown Majorana (anti-neutrinos are identical to neutrinos) or Dirac (anti-neutrinos are distinct from neutrinos) nature.

\subsection{Entropy, fugacity, and pressure}

The accurate thermodynamical quantities for fermions at all regimes can be calculated by evaluating numerically the relativistic Fermi-Dirac integrals for particle density $n$, energy density $e$, and pressure $P$ (Padmanabhan 2000, p. 216-217)

$$
\begin{aligned}
& n(T, Z)=\frac{4 \pi g_{\mathrm{s}}}{h^{3}} \int_{0}^{\infty} \frac{p^{2}}{Z^{-1} \exp (\epsilon / k T)+1} \mathrm{~d} p \\
& e(T, Z)=\frac{4 \pi g_{\mathrm{s}}}{h^{3}} \int_{0}^{\infty} \frac{p^{2} \epsilon}{Z^{-1} \exp (\epsilon / k T)+1} \mathrm{~d} p \\
& P(T, Z)=\frac{4 \pi g_{\mathrm{s}}}{h^{3}} \int_{0}^{\infty} \frac{p^{2}}{Z^{-1} \exp (\epsilon / k T)+1} \frac{1}{3} \frac{c^{2} p^{2}}{\epsilon+m c^{2}} \mathrm{~d} p
\end{aligned}
$$

where $g_{\mathrm{s}}$ is the number of distinct particle states, $\epsilon=$ $\sqrt{p^{2} c^{2}+m^{2} c^{4}}-m c^{2}$ is the particle energy, and $Z$ is the fugacity (the degeneracy parameter), capitalised here to distinguish from redshift $z$. The calculations in this paper have been performed with the software package Maple, version 10, in which the number of significant digits can be chosen. Here 14 digit precision has been used. 


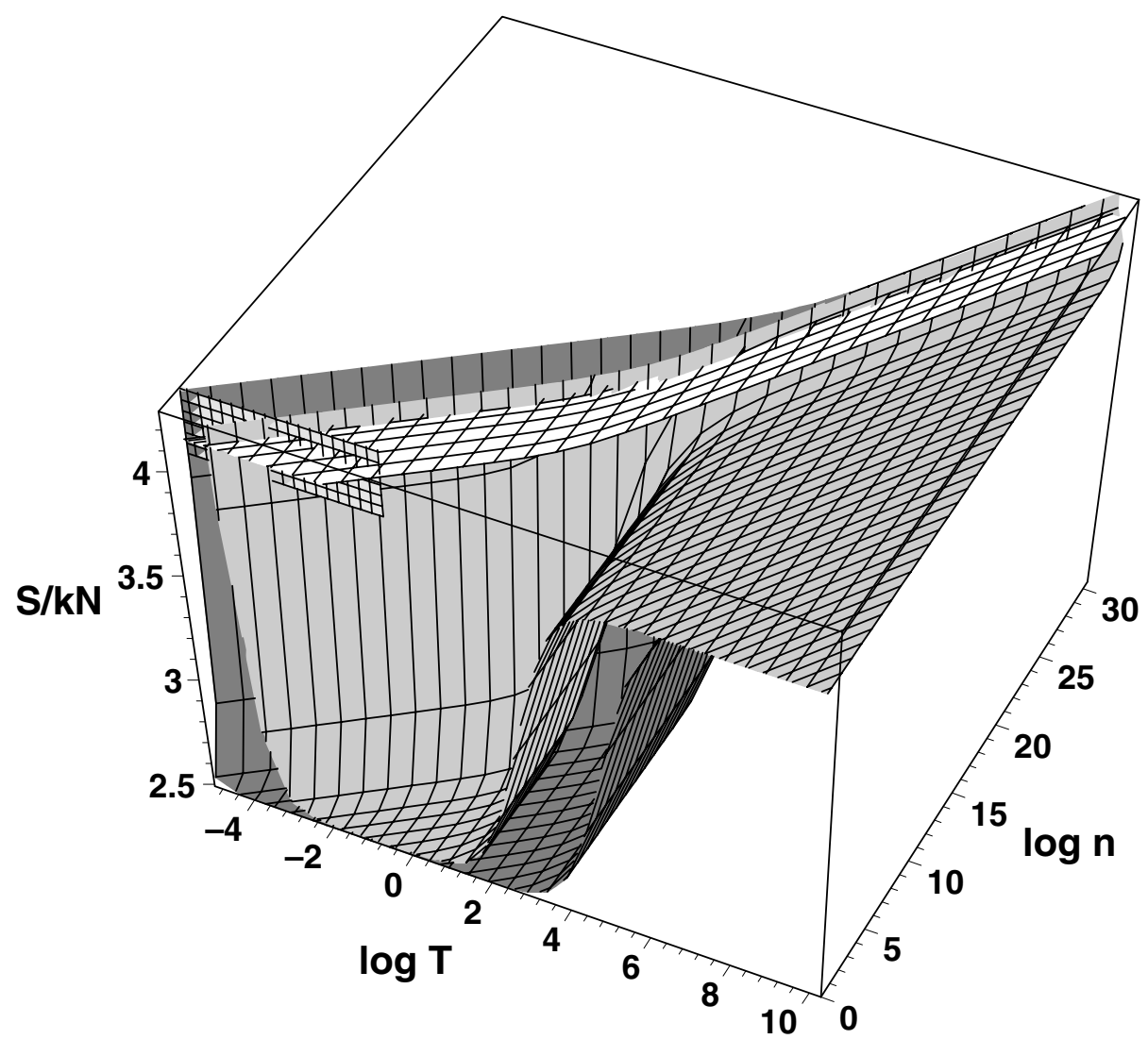

Fig. 1. The grey surfaces show the entropy per fermion $S / k N$ as a function of density and temperature $(\log n, \log T)$ for a neutrino mass of $m_{v}=0.1 \mathrm{eV}$ in light grey, and of $m_{v}=10 \mathrm{eV}$ in dark grey. The horizontal white band marks the constant entropy of 4.2018 expected from the hot Big Bang fermions. The vertical rectangle on the left shows the constant density of $n=56 \mathrm{~cm}^{-3}$ expected for each neutrino state at $z=0$. The grid lines on the entropy surfaces run parallel to either constant temperature or fugacity.
The adiabatic expansion of neutrinos since redshift $z=10^{10}$ means that the entropy per particle $S / k N$, a dimensionless number, has been constant since then. If the initial chemical potential is negligible, $S / k N$ amounts to 4.2018 for fermions, and 3.6016 for bosons (Peacock 1999, p. 277). The entropy per particle can be calculated from Eqs. (5)-(7) with the relationship $S / k N=(e+P) / n k T$, a function of $T$ and $Z$, which no longer depends on $g_{\mathrm{s}}$ and $h$. Calculating the implicit function $S / k N(T, Z)$ for a sufficiently wide range of $T$ and $Z$, we show in Fig. 1 its dependence on $n$ and $T$ over relevant cosmological values from high to low redshifts, for two representative neutrino masses, 0.1 and $10 \mathrm{eV}$. The grey parts, almost vertical in the back, correspond to degenerate states, with $n \propto T^{3 / 2}$ on the right in the relativistic regime, and $n \propto T^{3}$ on the left in the non-relativistic regime. The almost horizontal parts in the front of the $S / k N$ maps correspond to non-degenerate states not reached during the Universe expansion. The step passing from $S / k N=2.5$ to 4 around $\log T=2-4$ corresponds to the transition from the non-degenerate non-relativistic regime to the relativistic regimes. The position of the step does depend on the particle mass. Clearly, today's cosmological neutrinos with sub$\mathrm{eV}$ masses are much colder than the frequently quoted $1.95 \mathrm{~K}$ temperature valid for relativistic neutrinos.

Observing carefully the constant fugacity curves in Fig. 1, one notices that the adiabatic expansion from the relativistic to the non-relativistic regime increases the neutrino degeneracy, since the constant fugacity curves drop their entropy level. To make this point quantitative, we solve numerically for $T$ and $Z$ the pair of equations

$$
\frac{S}{k N}(T, Z)=4.2018, \quad n(T, Z)=56 \mathrm{~cm}^{-3},
$$

with a bisection search technique because the non-linear equation solver of Maple for floating-point number solutions fsolve based on a Newton-Raphson method fails in this case. The value of $n$ is a low value for today's neutrino density (choosing $n=112 \mathrm{~cm}^{-3}$ would reinforce our conclusions). We find that the temperature scales as the inverse of the mass, while $Z$ at $z=0$ is practically independent of the neutrino mass. We obtain for the present neutrino temperature,

$$
T_{v, 0}=1.614 \cdot 10^{-4}\left(\frac{m_{v}}{1 \mathrm{eV}}\right)^{-1} \mathrm{~K}, \quad \text { and }, \quad Z=16.50 .
$$

So between the creation of neutrinos at $z \approx 10^{10}$ and now, somehow fugacity has increased from 1 to 16.5 .

To show how fugacity behaves as function of redshift at constant entropy per particle, we ask Maple to solve numerically by interpolation the above quantities in Eqs. (5)-(7). The result is shown in Fig. 2. Fugacity increases from the early Universe to the present by a factor 16.5: massive neutrinos are substantially more degenerate now than at their decoupling epoch. This is independent of their mass if they are non-relativistic. When calculating the $Z(z)$ relationship for a range of entropies, we see that if for any reason entropy would increase instead of being strictly constant, fugacity would increase even more.

Associated to fermion degeneracy is the Fermi pressure contribution. To quantify this important factor for dynamics, again we ask Maple to evaluate numerically the integrals in Eqs. (5)(7), and to find by interpolation the pressure at constant specific entropy curve. In Fig. 3 the ratio of the full pressure to the perfect gas pressure $P_{c}=n k T$ is displayed. The additional quantum pressure with respect to a collisionless gas amounts to $5 \%$ at high redshifts, independent of the neutrino mass. At the transition from the relativistic to the non-relativistic regime, the redshift of which does depend on the mass, the quantum pressure contribution increases to $68 \%$, and becomes subsequently independent on the mass in the non-relativistic regime. Again any increase in specific entropy would increase pressure. 


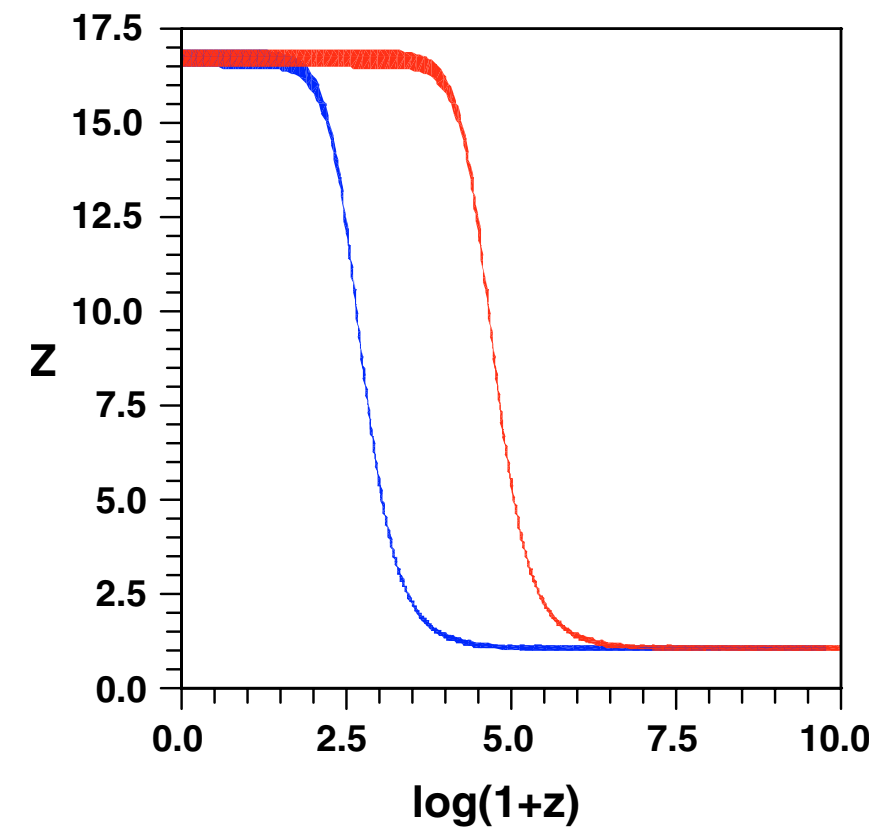

Fig. 2. Fugacity $Z$ as a function of redshift $z$ for constant comoving fermion density $n=56(1+z)^{3} \mathrm{~cm}^{-3}$ and constant entropy per fermion $S / k N$ in the range 4.2018 (lower limit of the bands) and 4.24 (upper limit of the bands) for $m_{v}=0.1 \mathrm{eV}$ (lower band), and $m_{v}=10 \mathrm{eV}$ (upper band).

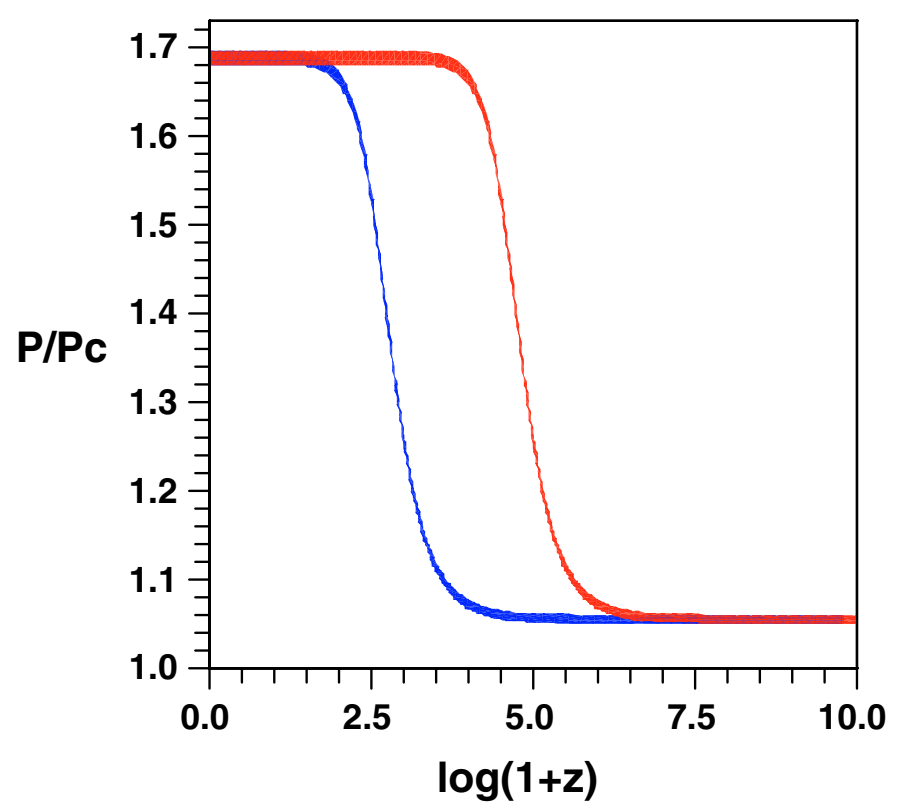

Fig. 3. Ratio of neutrino pressure $P$ to a perfect gas pressure $P_{\mathrm{c}}=n k T$, as a function of redshift $z$ for constant comoving fermion density $n=$ $56(1+z)^{3} \mathrm{~cm}^{-3}$ and constant entropy per fermion $S / k N$ in the range 4.2018 (lower limit of the bands) and 4.24 (upper limit of the bands) for $m_{v}=0.1 \mathrm{eV}$ (lower band), and $m_{v}=10 \mathrm{eV}$ (upper band).

Obviously this additional pressure is a substantial effect at low redshifts determining the clustering of neutrinos during the structure formation phase. The immediate prediction is that neutrinos should tend to accumulate less in bound structures than truly collisionless particles. However, dissipative effects in nonlinearities such as shocks may eventually falsify this prediction.

\subsection{Asymptotic estimates}

These previous results are exact down to the used 14-digit numerical precision. Let us now also derive intermediate states with asymptotic approximations, in part to estimate the errors made with this more traditional approach.

During the relativistic phase of expansion neutrinos cool proportionally to the photon temperature (Peebles 1993):

$T_{v, r}=\left(\frac{4}{11}\right)^{1 / 3} T_{\gamma}=\left(\frac{4}{11}\right)^{1 / 3} T_{0}(1+z)$,

where $T_{0}$ is today's CMB temperature of $2.73 \mathrm{~K}$. When the neutrino thermal energy equals its rest mass energy, neutrinos become non-relativistic. Then

$m_{v, r} c^{2}=3 k T_{v} \approx\left(\frac{4}{11}\right)^{1 / 3} 3 k T_{0}(1+z)$.

Then the critical temperature is

$T_{v, c}=\frac{m_{v} c^{2}}{3 k} \approx 3970\left(\frac{m_{v}}{1 \mathrm{eV}}\right) \mathrm{K}$

and the critical redshift

$1+z_{c}=1986\left(\frac{m_{v}}{1 \mathrm{eV}}\right)$

Below redshift $z_{c}$ temperature drops for adiabatic non-relativistic matter approximately as

$T_{v, n}=T_{v, c}\left(\frac{1+z}{1+z_{c}}\right)^{2} \approx 9.8 \cdot 10^{-4}(1+z)^{2}\left(\frac{m_{v}}{1 \mathrm{eV}}\right)^{-1} \mathrm{~K}$.

Thus the thermal speed of cosmological neutrinos amounts to

$v_{v}=\left(\frac{4}{11}\right)^{1 / 3} \frac{3 k T_{0}}{m_{\nu} c}(1+z) \approx 151(1+z)\left(\frac{m_{v}}{1 \mathrm{eV}}\right)^{-1} \mathrm{~km} \mathrm{~s}^{-1}$

(see Ringwald \& Wong 2004, for a similar derivation). With respect to the exact result Eq. (9), $T_{v, n}$ is overestimated by a factor 6.07, and $v_{v}$ by a factor 2.46 .

Owing to number conservation, the neutrino density $n_{v}$ varies in proportion of the volume

$n_{v}=n_{v, 0}(1+z)^{3}$.

The average phase space volume per neutrino is estimated as

$V_{v} \sim \frac{1}{n_{v}} \frac{4 \pi v_{v}^{3}}{3} \approx \frac{144 \pi}{11 n_{0}}\left(\frac{k T_{0}}{c}\right)^{3}$

which is independent of the neutrino mass and redshift, as long as neutrinos are non-relativistic. Compared with the elementary quantum volume $h^{3}$, we have,

$\frac{V_{v}}{h^{3}} \approx \frac{284}{n_{v, 0}} \approx 5.1\left(\frac{n_{v, 0}}{56}\right)^{-1}$

Neutrinos appear therefore rather close to be strongly degenerate, consistently with the previous accurate calculations. Therefore quantum effects must be considered with more attention. 


\section{Quantum effects}

\subsection{The paradox of collisionless identical particles in the quantum regime}

In view of the previous results, we are led to a paradox. On one side from cosmological conditions we find that neutrinos are fairly densely packed in phase space, so Pauli's exclusion principle should be applicable, while on the other hand in the usual view among cosmologists neutrinos should ignore each other since their particle-particle electro-weak coupling is very low. How can particles without interaction term interact?

Often in quantum mechanics one must use the idealisations of isolated, independent systems, although wave-functions formally extend over the whole space. This is justified (e.g., Cohen-Tannoudji et al. 2000, Chap. XIV, D, p. 1384) when the system wave-function is itself well separated from the outer world wave-functions. This is possible because particles are emitted not as plane waves extending uniformly over the whole space, but as wave-packets since any emission process takes a positive time-interval. Therefore the individual wave-functions consist of well defined square integrable functions with compact support, as required by quantum theory.

So if we picture individual neutrinos as localised moving wave-packets, they follow a classical free particle trajectory as long as the wave-packets are well separated. But when the wave-packet cores of identical particles overlap, then quantum interference must occur, blurring the possibility to distinguish the particles, because they must simultaneously follow Pauli's rule of anti-symmetrisation for fermions. Pauli's symmetrisation rules for fermions and bosons are independent of the particular particle kind and strength of the interaction potential.

All the interactions between elementary particle, nuclei, atoms, molecules, and even larger pieces of matter like superfluids, follow the Pauli symmetrisation rules: whenever particle wave-functions overlap, quantum mechanics is consistent with experiments at the condition of applying the wave-function symmetry postulate. Somehow the individual packets of identical particles which could be considered as independent when far apart, must form a symmetric or antisymmetric combined wave-function when they overlap. The speed of this (anti-)symmetrisation process has nothing to do with the electro-weak interaction, but just the quantum mechanical nature of identical particles.

Before discussing this paradox further, let us illustrate graphically with exact solutions of the Schrödinger equation how the wave-packets of two identical free fermions behave.

\subsection{Interaction between two identical free fermions}

It is not difficult to solve exactly the time-dependent Schrödinger equation,

$\mathrm{i} \hbar \frac{\partial}{\partial t} \Psi(\boldsymbol{x}, t)=H \Psi(\boldsymbol{x}, t)$,

for the Hamiltonian $H$ of two identical free fermions and illustrate graphically the behaviour of the most physical object representing a particle, the amplitude of the wave-function core. We illustrate below what happens to two spinless fermions in 3D space without explicit interaction potential. As initial condition we adopt Gaussian wave-packets because they allow to calculate all the results in closed form.

As derived in standard quantum mechanics, the full solution of the single free particle Schrödinger equation in 3D Cartesian space reads (with units normalised conveniently for our cosmological neutrino context to $[l]=1 \mathrm{~cm},[\mathrm{~m}]=0.1 \mathrm{eV}$, and $[t]=1.7 \times 10^{-7} \mathrm{~s}$, which allows to set $\left.\hbar / m=1\right)$ :

$$
\Psi(\boldsymbol{x}, t)=\frac{1}{(2 \pi \mathrm{i} t)^{3 / 2}} \int \exp \left(\mathrm{i} \frac{(\boldsymbol{x}-\boldsymbol{y})^{2}}{2 t}\right) \Psi(\boldsymbol{y}, 0) \mathrm{d}^{3} y .
$$

Thus finding how an arbitrary initial wave-function $\Psi(\boldsymbol{x}, 0)$ evolves is just a matter of calculating a triple convolution integral at any wished time $t$. For particular initial $\Psi(x, 0)$ such as Gaussian packets the integral can be completely expressed in closed form, which we manipulate below with Maple.

When describing two independent distinguishable particles, the full wave-function takes the form

$\Psi\left(x_{1}, x_{2}, t\right)=\Psi_{1}\left(x_{1}, t\right) \Psi_{2}\left(x_{2}, t\right)$,

for which the time evolution is just the product of the single particle solution Eq. (20).

But this is only a very particular form of all the wavefunctions $\Psi\left(\boldsymbol{x}_{1}, \boldsymbol{x}_{2}, t\right)$ that the Schrödinger equation for two particles can describe. Functions that cannot be separated as a product of single particle wave-function describe entangled states. In particular two identical particles must follow either a symmetric or anti-symmetric wave-function upon the exchange of the particles. These read

$\Psi_{ \pm}\left(x_{1}, x_{2}, t\right)=\frac{1}{\sqrt{2}}\left[\Psi_{1}\left(x_{1}, t\right) \Psi_{2}\left(x_{2}, t\right) \pm \Psi_{1}\left(x_{2}, t\right) \Psi_{2}\left(x_{1}, t\right)\right]$

where the + sign applies to symmetric functions and the - sign to antisymmetric functions. The time evolution of such an entangled pair of wave-functions can also be calculated exactly in closed form by forming the product and sum of single particle solutions. The explicit formula is too long to show here, but can be easily manipulated with Maple or similar software tools.

Although the wave-function of two particles is 6dimensional and complex, when the particle centres of mass and velocity are set at the origin we can focus the attention on the function $\Psi_{-}(\boldsymbol{x},-\boldsymbol{x}, t)$ since any measurement of a particle at location $x$ will constrain the position of the other particle at position $-\boldsymbol{x}$, since we assumed to know the centre of mass. $\left|\Psi_{-}(\boldsymbol{x},-\boldsymbol{x}, t)\right|^{2}$ describes the probability of finding one particle at position $\boldsymbol{x}$ and the other one at $-\boldsymbol{x}$.

Below we show graphically the normalised value $\left|\Psi_{-}(\boldsymbol{x},-\boldsymbol{x}, t)\right|^{2}$ in the plane $z=0$ for the initial condition in the centre of mass and velocity frame of two fermions represented initially by a Gaussian wave-packet of unit half-width:

$\Psi_{1}(\boldsymbol{x}, 0)=\exp (i \boldsymbol{k} \cdot \boldsymbol{x}) \exp \left[-\left(\boldsymbol{x}-\boldsymbol{x}_{0}\right)^{2} / 2\right]$.

The initial position of particle 1 is $\boldsymbol{x}_{1}(0)=\{-5,-2,0\}$, and the initial momentum is $\boldsymbol{k}_{1}=\left\{k_{x}, 0,0\right\}$. The initial position of particle 2 is symmetric about the origin in order to put the centre of mass and velocity at the origin. The momentum is therefore all in the component $k_{x}$, which is not directed toward the centre of mass in order to illustrate a generic interaction with non-zero impact parameter.

Here we show three different representative values of the momentum $k_{x}$ illustrating the classical $\left(k_{x}=6\right)$, semi-classical $\left(k_{x}=2\right)$, and quantum regimes $\left(k_{x}=2 / 3\right)$. This should suffice for our purpose of illustrating the exchange interaction of free identical particles.

In Fig. 4 we see the two wave-packets individually spreading slowly due to their high momentum $k_{x}=6$. Despite that, 

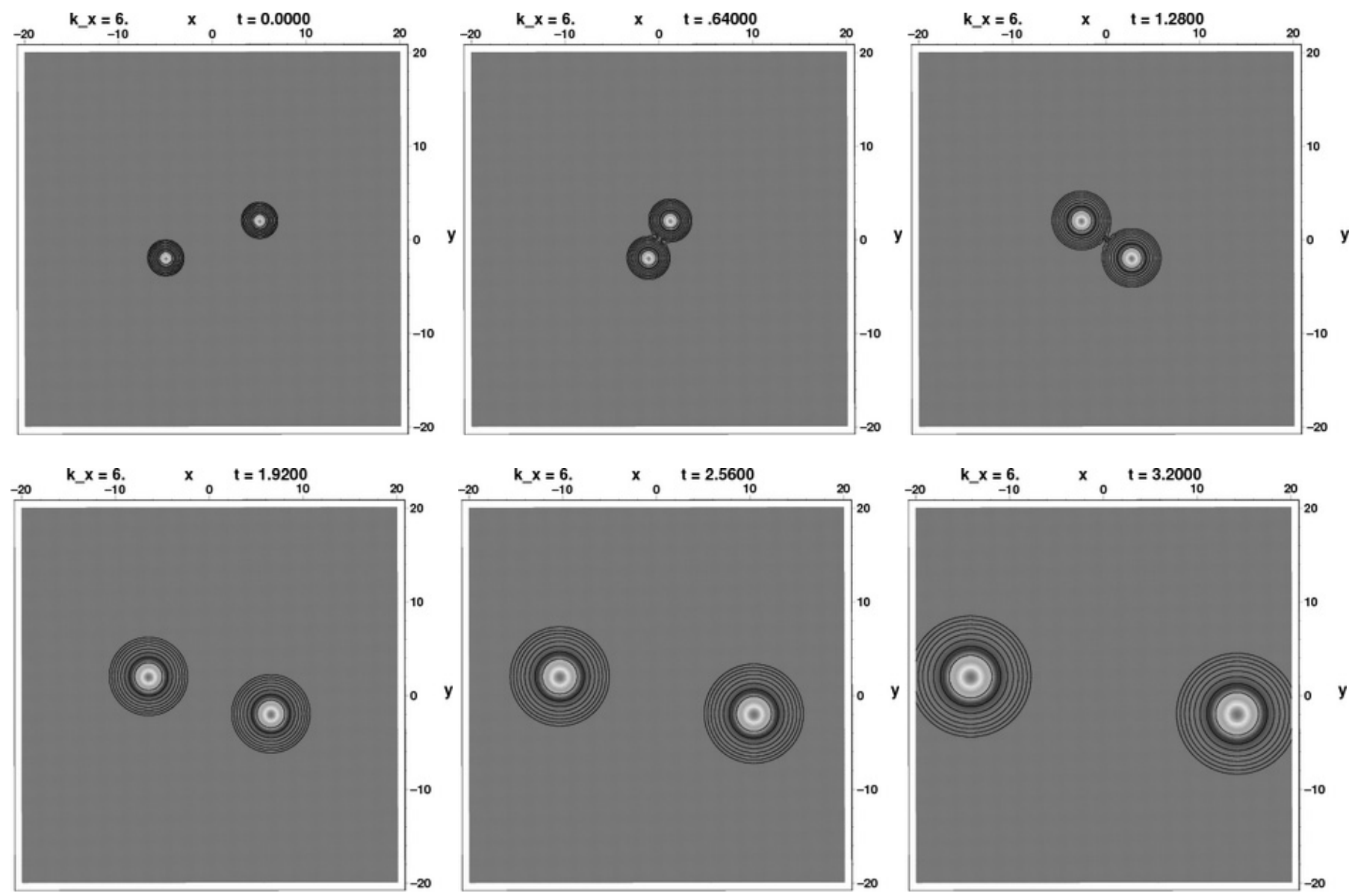

Fig. 4. Interaction of two free fermions in the classical regime starting initially in opposite directions and horizontally $\left(k_{x}=6, k_{y}=0, k_{z}=0\right)$. Shown is the normalised squared norm $\left|\Psi_{-}(\boldsymbol{x},-\boldsymbol{x}, t)\right|^{2}$ in the plane $(x, y, 0)$.

for a while the packets strongly overlap, subsequently they recover their localised wave-functions. In such a case the individual packets behave as expected for classical particles: they move in straight line and preserve their identity. Although Pauli's principle applies, it has no practical consequence asymptotically. These two particles can be approximated as distinct again at later times.

In Fig. 5 we see that at lower momentum, $k_{x}=2$, two wavepackets interfere much more strongly. The antisymmetric wavefunction always vanishes at the mass centre. The size of this exclusion region around the origin grows at lower velocities. The interference pattern presents elongated wave crests which are inclined with respect to the original particle momentum directions. A plane wave seems to be building-up in a direction slanted with respect to the particles directions. Not only the positions are strongly mixed in a single structure, but the momentum becomes also mixed. After a while the individual wave-packets representing the classical localised particles succeed to partly reform, but a remaining coma of entangled states remains around the origin.

At still lower relative momentum, $k_{x}=2 / 3$, quantum effects become dominant. In Fig. 6 the two particles initially distinct rapidly loose their identity and form a single anti-symmetrical wave-packet which spreads around the origin with waves again reflecting an intermediate direction of propagation with respect to the initial particle momentum. Clearly in this case the probability of finding a neutrino somewhere else than along the initial straight trajectories is strongly enhanced by the quantum interferences. The classical approximation of straight trajectories is no longer valid, and the initially distinct particles are entangled forever. In the quantum regime a clear dispersive behaviour occurs with marked asymmetric past-future asymptotic states.

The same experiments have been repeated for bosons. The only notable difference is that the wave-functions are always positive at the origin, and even take there maximum values in the quantum case: this corresponds to the Bose-Einstein condensation. In contrast, the fermionic wave-functions always vanish exactly at the origin, which means that a volume of space around the origin is hardly available to the particles. Fermi pressure is a consequence of confining the particle energy in a reduced volume.

\subsection{Interaction between $\mathrm{N}$ identical free fermions}

The problem of describing statistically $N$ identical bosons or fermions with or without interactions has been solved very early in the history of quantum mechanics (Uhlenbeck \& Gropper 1932), but is much more difficult to illustrate graphically than the 2 particle case. What is clear is that in the limit of vanishing particle interaction, the bosonic or fermionic statistical behaviour tends toward classical Maxwell-Boltzmann statistic only at high temperature. At sufficiently low temperature the statistical behaviour tends toward the Bose-Einstein and Fermi-Dirac statistics respectively.

An interesting aspect developed for example by Huang (1964, chap. 10), is that the first quantum correction to a classical perfect gas, which is appropriate for our cosmological neutrino context, caused purely by the bosonic or fermionic nature of the particles is mathematically equivalent to an particle-particle 

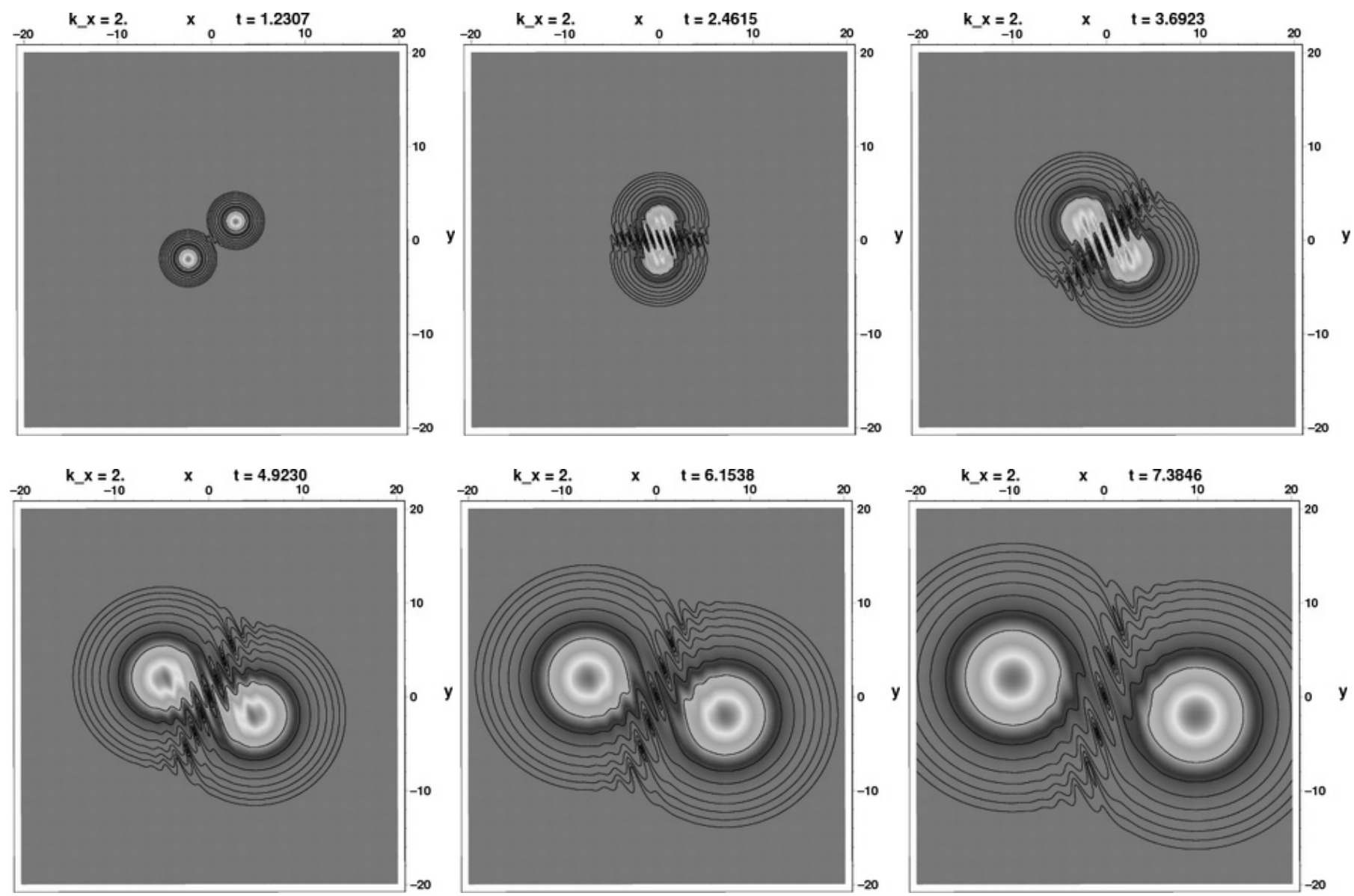

Fig. 5. As in Fig. 4, but in the semi-classical regime $\left(k_{x}=2\right)$.

interaction potential, with the peculiarity to depend also on temperature $T$,

$\phi\left(\boldsymbol{x}_{i}-\boldsymbol{x}_{j}\right)=-k T \log \left[1 \pm \exp \left(-2 \pi \frac{\left|\boldsymbol{x}_{i}-\boldsymbol{x}_{j}\right|^{2}}{\lambda_{\mathrm{dB}}^{2}}\right)\right]$.

Figure 7 shows that the pseudo potential is repulsive for fermions and attractive for bosons. The potential becomes important $(\phi \sim$ $k T$ ) at a distance slightly below the de Broglie thermal wavelength $\lambda_{\mathrm{dB}}=h / \sqrt{2 \pi m k T}$.

This is consistent with the rule that quantum effects become important when thermalised particles, are less distant in the classical description than $\lambda_{\mathrm{dB}}$. The potential permits to derive an effective interaction cross-section, and from there the time required for particle to become entangled. We will refer to this time as the entanglement time-scale. If the particles were classical we would call this a collision time-scale, that would determine a relaxation time.

If we adopt a classical description of neutrinos moving in such a potential, clearly a strong deflection of trajectory occurs whenever $\phi / k T \sim 1$, which means also that $\left|\boldsymbol{x}_{i}-\boldsymbol{x}_{j}\right| \sim \lambda_{\mathrm{dB}}$, so the interaction cross-section reads $\sigma \sim \pi \lambda_{\mathrm{dB}}^{2}$. Thus the first order quantum correction to a classical description of semi-degenerate neutrinos introduces an interaction potential producing "entanglement" with a characteristic time-scale given by

$$
\begin{aligned}
\tau \approx \frac{1}{n_{v} \sigma v_{v}} & \approx \frac{m_{v}^{3 / 2} \sqrt{k T}}{n_{v} h^{2}} \\
& \approx 1.3 \times 10^{-8}(1+z)^{-2}\left(\frac{n_{v, 0}}{56}\right)^{-1}\left(\frac{m_{v}}{1 \mathrm{eV}}\right)^{-1} \mathrm{~s},
\end{aligned}
$$

using the asymptotic relations for $T, n$, and $v$ given in Sect. 3.3. This yields a very short time-scale $\ll 10^{-6} \mathrm{~s}$ for any reasonable present day and past cosmological neutrinos. Although this derivation is not consistent since quantum particles do not follow classical trajectories, at least $\tau$ characterises the entanglement time-scale between neighbour neutrinos.

\subsection{Decoherence time}

In the decoherence theory (Zeh 1970, 2005; Zurek 2002, 2003), classical physics emerges from the much larger sensitivity of the entangled states in the density matrix $\Psi(x) \Psi^{*}\left(x^{\prime}\right), x \neq x^{\prime}$, to the interferences coming from the outer world than the diagonal states $\Psi(x) \Psi^{*}(x)$. Particles become localized classical objects because the uncorrelated perturbations from the outside world prevent the wave-function to spread. The decoherence time $\tau_{D}$ for a quantum system considered over a distance $\Delta x$ to loose quantum correlations $\Psi(x) \Psi^{*}\left(x^{\prime}\right), \Delta x<\left|x-x^{\prime}\right|$, is related to the relaxation time $\tau_{R}$ induced by the outer world, and to $\lambda_{\mathrm{dB}}$ :

$\frac{\tau_{\mathrm{D}}}{\tau_{\mathrm{R}}}=\left(\frac{\lambda_{\mathrm{dB}}}{\Delta x}\right)^{2}$

Thus the decoherence time is inversely proportional to the square of the considered region. Obviously, to quantify decoherence we must identify the fastest process that perturb or "relax" neutrinos.

Let us make a best effort to find the shortest possible $\tau_{R}$ that cosmological neutrinos might be subject to. If we discard weak interaction processes (cf. Eq. (1)), we only have gravitational deflection available. 

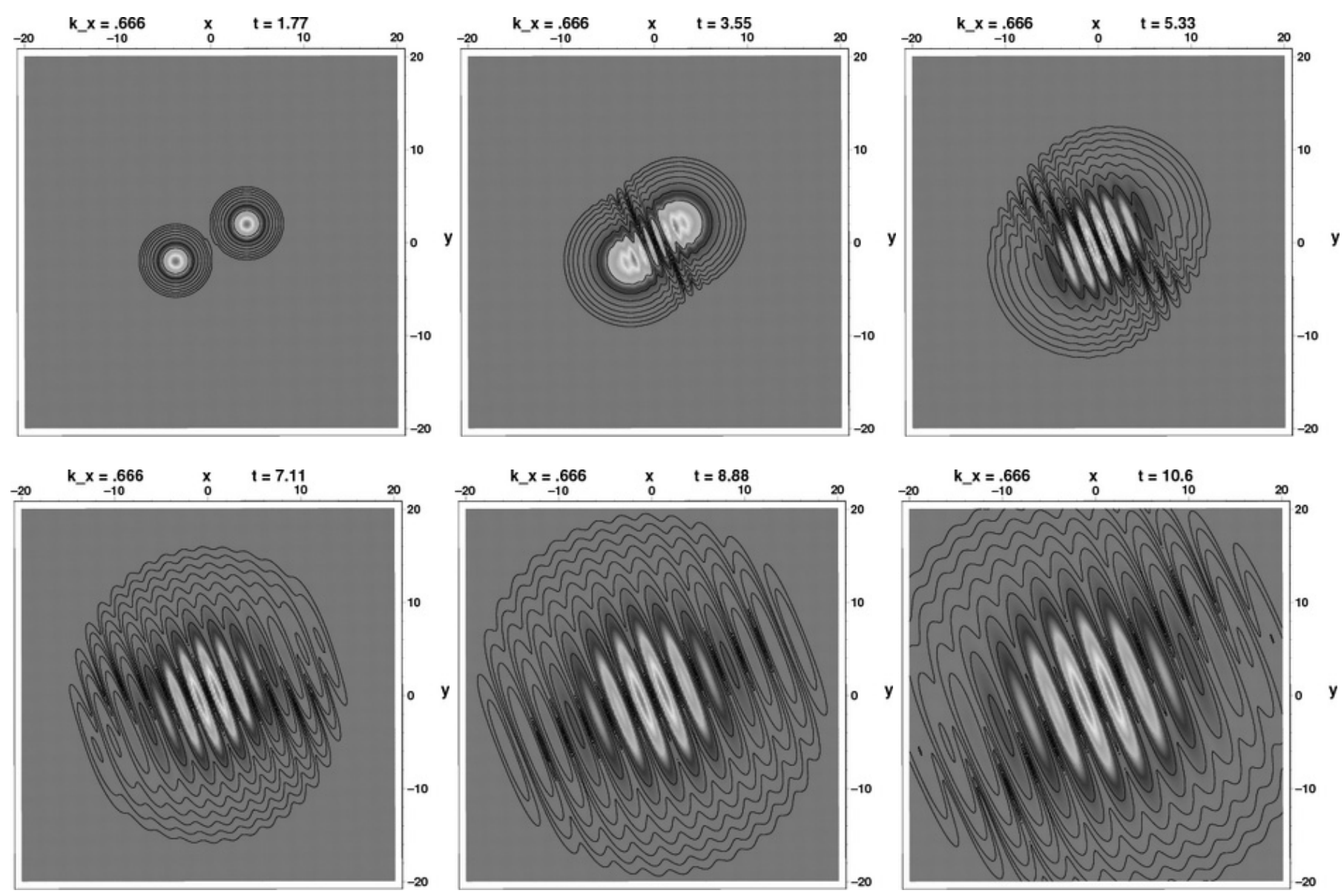

Fig. 6. As in Fig. 4, but in the quantum regime $\left(k_{x}=2 / 3\right)$.

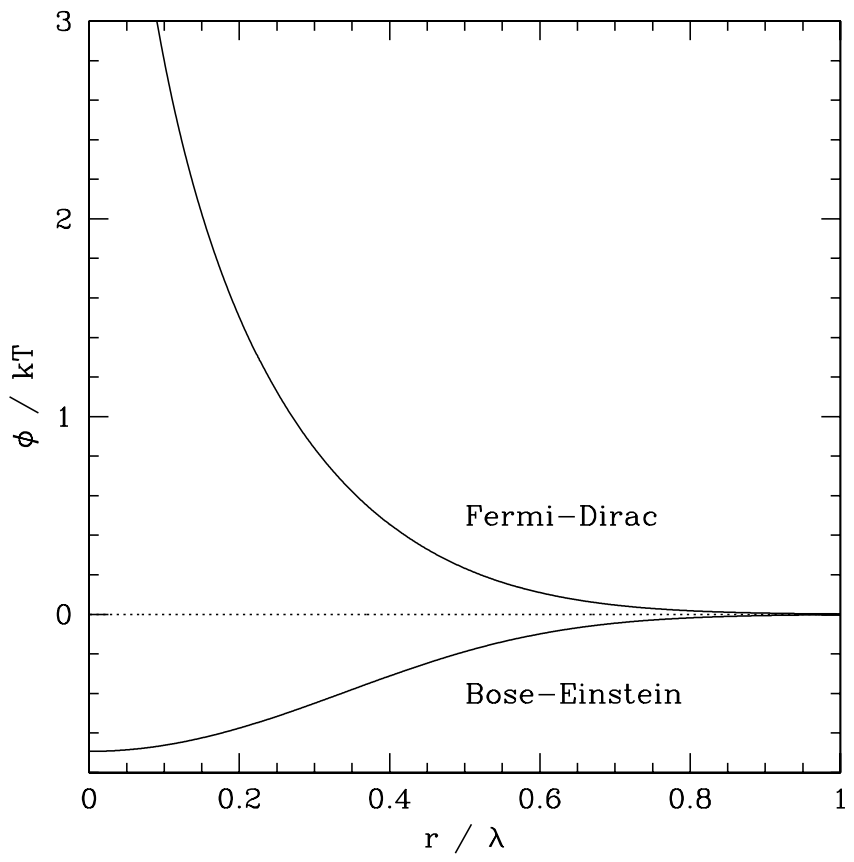

Fig. 7. Effective particle-particle interaction potential $\phi$ for a perfect gas of fermions (top curve, repulsive potential), or bosons (bottom curve, attractive potential) in first order of quantum correction, as a function of distance $r$ in unit of the de Broglie thermal wavelength $\lambda_{\mathrm{dB}}$.

Suppose we have a density $n$ of bound gravitational objects of mass $M$. The gravitational cross-section for neutrinos at velocity $v_{v}$ is $\sigma=\pi G^{2} M^{2} / v_{v}^{4}$, and the timescale for deflecting significantly neutrinos by these objects is:

$\tau_{\mathrm{R}}=\frac{1}{n \sigma v_{v}}=\frac{v_{v}^{3}}{\pi G^{2} n M^{2}}$.

Thus at constant mass density $n M$ more massive objects deflect faster than light objects. Galaxies seem the most promising deflectors, because stars are mostly bound in galaxies and amount to only a fraction of their mass, while galaxy clusters contain a small fraction of the galaxies.

Plugging in plausible numbers for galaxies $\left(n \approx 10^{-74} \mathrm{~cm}^{-3}\right.$, $M \approx 10^{45} \mathrm{~g}$ ), and $v_{v} \approx 10^{8} \mathrm{~cm} \mathrm{~s}^{-1}$, we obtain $\tau_{\mathrm{R}} \sim 10^{22} \mathrm{~s}$. Therefore for $\tau_{\mathrm{D}}$ to be less than the entanglement time $<10^{-6} \mathrm{~s}$, we need to consider regions larger than

$\Delta x=\lambda_{\mathrm{dB}} \sqrt{\frac{\tau_{\mathrm{R}}}{\tau_{\mathrm{D}}}} \approx 10^{13} \mathrm{~cm}$,

in order to begin to use classical physics. In such a region of $\mathrm{AU}$ dimension we have $\sim 10^{40}$ neutrinos that behave as a fermionic ensemble. Since the relaxation time $\sim 10^{22} \mathrm{~s}$ is much larger than the neutrino age $\sim 10^{17} \mathrm{~s}$, most neutrinos that would be localized by hypothetical detectors would still be entangled with neutrinos at cosmic distances apart. The ratio of these times, $10^{-5}$, gives the fraction of neutrinos that have been perturbed by the gravitational attraction of a galaxy. After $10^{5}$ entanglement times of $<10^{-6} \mathrm{~s}$, each neutrino has a large probability to be also entangled with perturbed neutrinos, thus propagating the coherence loss. 


\section{Discussion}

\subsection{The status of quantum physics}

The quantum physics rules are so strange that it took a long time for the physicist community to make a standard view about its principal aspects. Today several points are not completely resolved, such as the meaning of the measurement process, or the rôle of time in a relativistic quantum theory of several particles (Penrose 2005). Quantum gravity is still in the work.

However in the last decade many new experiments have confirmed the strange rules of quantum mechanics. The EinsteinPodolsky-Rosen (EPR) paradox has been confirmed, two entangled particles can fly apart over long distances and preserve their mutual correlation alive even when one of the particle is subject to measurement. In recent experiments (Tittel et al. 1998) the photon entanglement has been preserved over distances of the order of $10 \mathrm{~km}$ throughout a complicated network of telecommunication optical fibres.

\subsection{Hanbury Brown and Twiss (HBT) effect and the quantum decoherence}

At the root of quantum optics, there is a fine example of exchange symmetry interaction operating on otherwise interactionless particles: photons. This effect was first investigated by Hanbury Brown and Twiss (Hanbury Brown \& Twiss 1954) with the now famous intensity interferometer (Hanbury Brown 1974). The effect could also be performed at the laboratory scale (Morgan \& Mandel 1966) and was an important step in the development of quantum optics. In the Hanbury Brown and Twiss experiment, independent incoherent photons emitted all over the surface of a star $\left(D \sim 10^{9} \mathrm{~m}\right)$ at distinct times are collected on Earth by two distinct telescopes separated by macroscopic distances $\left(d \sim 10^{2} \mathrm{~m}\right)$. The collected distinct photons arriving in the two detectors within a photon coherence time are verified to be correlated in time. Despite being particles without explicit coupling term and initially incoherent, photons sufficiently close in phase space (they are very close in momentum space) succeed to bunch, i.e., to exchange momentum. This is an example of a partial Bose-Einstein condensation.

The fermion effect corresponding to the HBT experiment has been confirmed recently for electrons in laboratories (Henny et al. 1999; Kiesel et al. 2002). Such experiments are complicated by the fact that the repulsive electro-magnetic interaction term of electrons must be cancelled by either an atom lattice, or a sufficiently large distance between electrons. In these experiments, electrons overlapping their wave-functions are found to be anti-correlated, which is at the root of the degeneracy pressure in fermionic gases. Better suited particles are neutrons, for which the HBT effect has also been verified recently (Iannuzzi et al. 2005).

The important point of these verified phenomena is to show that identical particles interact also through their exchange symmetry, and that the speed of this kind of interaction, not explicit in the Hamiltonian, is in practice determined by the speed at which the core of the wave-functions overlap significantly.

If we take the strong overlap of the particle wave-functions as the principal requirement for particles to be said interacting, and the weak overlap of many uncorrelated wave-functions as the regime during which the decoherence due to the rest of the world acts, we can understand why our common experience of the world is not a fully entangled structure (Penrose 2005), but the classical world that we know, where spatially distinct regions and classical devices can be assumed to exist. Correlations between particles are constantly lost by decoherence and built by wave-function overlaps. The macroscopic result for multiparticle systems is equivalent to a relaxation toward a thermal state (Gemmer et al. 2004).

Therefore if we apply these considerations to cosmological neutrinos, these must overlap their wave-function very frequently $\left(\ll 10^{-6} \mathrm{~s}\right)$, and each "interaction" produces a partial entanglement of the wave-functions. Quickly neutrinos get entangled to a growing number of other neutrinos which together form a spreading entangled wave-function. Any perturbation or "observation" of a given neutrino entangled with others concerns then all the other neutrinos. Everything happens as if the detected neutrinos had exchanged momentum with the others, like classical particles in usual interactions do.

\section{Conclusions}

In view of the solidity of quantum mechanics and the likely sub$\mathrm{eV}$ mass of neutrinos, we arrive to the conclusion that quantum effects play an dominant rôle for cosmological neutrinos. Despite being almost interactionless, these neutrinos form a nonlocalisable ensemble of entangled particles subject to Pauli's principle. The weakness of their interactions with the outer world is precisely the key factor that prevents them to decohere faster than they become entangled. The only way to justify a classical description of neutrinos following the collisionless Boltzmann's equation would be to find a perturbative process that would decohere all of them in much less than $10^{-6} \mathrm{~s}$. But then this perturbative process should presumably be included in the neutrino dynamics.

With the most accurate description of particles, the wavefunction, we have illustrated explicitly how a pair of initially localised neutrinos becomes entangled fast due only to their antisymmetry; quickly the localised wave-packets become a single fully non-localised object. In practice this means that more neutrinos act rapidly as an ensemble that must be treated with the standard quantum statistics tools. Since, except for antisymmetry, no interaction potential is significant, the appropriate description at cosmological scales is simple, the one of a semidegenerate perfect fermion gas.

For structure formation, the Fermi pressure contribution has little importance as long as the Universe is homogeneous, since only pressure gradients play a dynamical rôle. At redshifts $z \gg$ $10^{5}$ the Fermi over-pressure amounts to $5 \%$ of the kinetic pressure. But at redshifts $z \ll 10^{4}$ in the non-relativistic regime, during the non-linear structure formation epoch, the over-pressure increases to $68 \%$. Then a Fermi gas of massive neutrinos is substantially different from a collisionless gas. Not only the velocity dispersion remains then isotropic in a tightly coupled gas, but since collisional fluids are able to shock due to the non-linear convective term in the fluid equations, the evolution of the combination of neutrino gas, dark matter, and baryons in the today Universe may turn out to be complex. Shocks typically increase entropy, degeneracy, and therefore pressure, as seen in Sect. 3. It would be interesting to determine how far neutrinos could violate the phase space density conservation by dissipative processes, especially when mixed with baryons.

The extension of these results to other dark matter particle candidates resulting from the Big Bang should be straightforward. Relativistic fermions, degenerate or not, can not contribute significantly to matter clustering. Usual heavy cold dark matter fermions, such as neutralinos, are assumed much less dense and 
much heavier than neutrinos, in which case phase space degeneracy may remain small and decoherence fast, allowing the use of the collisionless fluid approximation.

Acknowledgements. We thank particularly Nicolas Gisin, Thanu Padmanabhan, and Jack Steinberger for sharing their point of views and insight of physicists. We have appreciated private discussions with Joel Primack and Ben Moore about the cosmologist view. We enjoyed discussions with Silvia Pascoli and Mario Campanelli at CERN about recent experimental results on neutrino physics. We thank our colleagues André Maeder and Denis Puy for lively discussions too. This work has been supported by the Swiss National Science Foundation.

\section{References}

Cohen-Tannoudji, C., Diu, B., \& Laloë, F. 2000, Mécanique quantique (Paris: Hermann)

Dolgov, A. D. 2002, Phys. Rep., 370, 333

Gemmer, J., Michel, M., \& Mahler, G. 2004, Quantum Thermodynamics, Lecture Notes in Physics, 657 (Berlin: Springer-Verlag)

Dirac, P. A. M. 1926, Proc. R. Soc. A, 112, 661

Fermi, E. 1926, Zeitschrift für Physik, 36, 902

Hanbury Brown, R., 1974, The Intensity Interferometer (London: Taylor \& Francis)

Hanbury Brown, R., \& Twiss, R. Q. 1954, Phil. Mag. 45, 663

Henny, M., Oberholzer, S., Strunk, C., et al. 1999, Science, 284, 296

Huang, K. 1964, Statistical Mechanics (New York: Wiley \& Sons, Inc.)
Iannuzzi, M., Orecchini, A., Sacchetti, F., Facchi, P., \& Pascazio, S. 2005, [quant-ph/0509131]

Kiesel, H., Renz, A., \& Hasselbach, F. 2002, Nature, 418, 392

Klypin, A., Holtzman, J., Primack, J., \& Regos, E. 1993, ApJ, 416, 1

Morgan, B. L., \& Mandel, L. 1966, Phys. Rev. Lett., 16, 1012

Padmanabhan, T. 2000, Theoretical Astrophysics, Vol. I (Cambridge Univ. Press)

Padmanabhan, T. 2002, Theoretical Astrophysics, Vol. III (Cambridge Univ. Press)

Pauli, W. 1925, Z. Phys., 31, 765

Peacock, J. A. 1999, Cosmological Physics (Cambridge Univ. Pres)

Peebles, P. J. E. 1993, Principles of Physical Cosmolog (Princeton Univ. Press)

Penrose, R. 2005, The Road to Reality, A Complete Guide to the Laws of the Universe, Knopf A.A. (New York: Borzoi Books)

Primack, J. R., \& Gross, M. A. K. 2000, in Current Aspects of Neutrino Physics, ed. D. O. Caldwell (Berlin: Springer), 287

Ringwald, A., \& Wong, Y. 2004, JCAP, 12, 005

Strack, P., \& Burrows, A. 2005, Phys. Rev. D, 71, 3004

Tegmark, M., Vilenkin, A., \& Pogosian, L. 2005, Phys. Rev. D, 71, 3523

Tittel, W., Brendel, J., Gisin, B., Herzog, T., Zbinden, H., Gisin, N. 1998, Phys. Rev. A, 57, 3229

Tremaine, S., \& Gunn, J. E., 1979, Phys. Rev. Lett., 42, 407

Uhlenbeck, G. E., \& Gropper, L. 1932, Phys. Rev., 41, 79

Weinberg, S. 1972, Gravitation and Cosmology (New York: Wiley \& Sons, Inc.) Zeh, H. D. 1970, Found. Phys., 1, 69

Zeh, H. D. 2005, Séminaire Poincaré, 2, 1 [quant-ph/0512078]

Zurek, W. H. 2002, Los Alamos Science, 27, 2

Zurek, W. H. 2003, Rev. Mod. Phys., 75, 715 